\title{
SPECTRUM OF OVARIAN LESIONS IN A TERTIARY CARE HOSPITAL
}

\author{
Kiran Sapru1, Letha P2, Devi B3
}

${ }_{1}^{1}$ Assistant Professor, Department of Pathology, Azeezia Institute of Medical Sciences, Kollam, Kerala, India. ${ }^{2}$ Associate Professor, Department of Pathology, Azeezia Institute of Medical Sciences, Kollam, Kerala, India. ${ }^{3}$ Assistant Professor, Department of Pathology, Azeezia Institute of Medical Sciences, Kollam, Kerala, India.

\section{ABSTRACT}

\section{BACKGROUND}

Ovarian tumours are the 7 th most common cause of mortality. About $80 \%$ ovarian lesions are benign. The tumours that occur in the ovary are usually primary and metastatic cases have occasionally been reported. We wanted to analyse the spectrum of lesions in ovary.

\section{METHODS}

This is a retrospective study done in 3228 women who came to our hospital during the period January 2017-December 2018. All patients who had undergone ovariotomy were included in the study irrespective of whether hysterectomy was done or not.

\section{RESULTS}

A total of 3228 cases were examined of which $47.5 \%$ cases were normal. Non-neoplastic lesions constituted 38.7\%. Among the neoplastic lesions, $12.7 \%$ cases were benign and $0.86 \%$ were malignant.

\section{CONCLUSIONS}

Ovarian tumours include a wide spectrum of lesions. Emergence of borderline tumours with prognostic difference from benign and malignant counterparts has added a new dimension to the research in field of ovarian tumours. Correlation of clinical and radiological findings has assisted the pathologist to arrive at a definite conclusion in almost all cases. Even then, we may require the help of modern techniques like IHC and hormonal studies. Understanding in detail about the different lesions in the ovary, their prognostic implications and differences in the treatment is also crucial in diagnosis. A proper, complete and definite diagnosis helps the clinician to initiate the best conservative treatment available.

HOW TO CITE THIS ARTICLE: Sapru K, Letha P, Devi B. Spectrum of ovarian lesions in a tertiary care hospital. J. Evolution Med. Dent. Sci. 2019;8(19):1559-1562, DOI: 10.14260/jemds/2019/346

\section{BACKGROUND}

Ovary is unique in the spectrum of lesions that can arise from it ${ }^{1}$. Tumours can arise from the wide variety of cells in ovary. This is due to complex structure of ovary as well as its specific functions ${ }^{1}$. Most common lesions encountered in ovary are functional or benign cysts and tumours. Ovarian neoplasm is usually detected towards late stage and are become large in size because of their presentation with mild symptoms ${ }^{2}$. Majority of the ovarian neoplasms are benign, although it's one of the most usual sites for primary neoplasm. But literatures of metastasis to ovary have also occasionally reported. Diagnosing ovarian tumours on the basis of clinical and gross characters may be challenging but provide important clues in arriving at diagnosis. Sex cord stromal tumours are mostly unilateral whereas metastatic tumour usually have a bilateral presentation. Most of the benign surface epithelial tumours have a cystic presentation. On gross examination, solid tumours with papillary projections may favour malignancy. However microscopic features of by these tumours help in arriving at an accurate diagnosis ${ }^{3}$.

'Financial or Other Competing Interest': None.

Submission 21-03-2019, Peer Review 23-04-2019,

Acceptance 30-04-2019, Published 13-05-2019.

Corresponding Author:

Letha $P$,

Associate Professor, Department of Pathology,

Azeezia Institute of Medical Sciences,

Kollam, Kerala, India.

E-mail: drlethapadmom@gmail.com

DOI: $10.14260 /$ jemds/2019/346

\section{(c) (i) $\$$}

Nonneoplastic lesions of the ovary may presents as a pelvic mass and are can also be associated with abnormal hormonal levels, simulating an ovarian neoplasm creating a dilemma regarding diagnosis on clinical examination, at time of surgery, or on pathologic examination. Most of these lesions are seen during the reproductive age group and can be associated with infertility 3 . These lesions must be properly identified and treated, so that conservative treatment can be done wherever possible, thereby avoiding unnecessary oophorectomy.

Low-grade serous carcinoma belongs to the Classic type I tumours is, which is usually associated with KRAS and BRAF mutations. These patients don't have Tp 53 mutation. Highgrade serous carcinoma belongs to the category of type II tumours characterized by a high level of genetic instability and TP53 mutations is seen in nearly almost all cases and are highly unstable. These tumours are rapidly growing, very aggressive and are usually diagnosed at an advanced stage. Type 1 and type 2 tumours develop by means of separate pathways. Borderline serous tumour/atypical proliferative tumours is the precursor of low-grade serous carcinoma. Non-invasive serous tumours with micropapillary pattern are probably be associated with synchronous or metachronous invasive disease 4 .

Only a few cases have been reported regarding development of high-grade serous carcinoma from a lowgrade serous carcinoma as most of the low-grade serous carcinomas develop along a different pathway that is quite unambiguous from the pathway of their high-grade counterparts. Favoured cell of origin for serous neoplasia is ovarian surface mesothelium. It has been found that as a result of ovulatory trauma, there can be Mullerian 
metaplastic change to a tubal epithelial type, with further neoplastic transformation related to mutational events. Almost all cases of serous epithelial tumours arise generally from metaplastic change of the surface mesothelium. According to recent studies in women who are having BRCA mutations, an alternative tubal origin is being put forward as an important source of high-grade serous carcinomas which was based on the evaluation of risk reducing salpingooophorectomy specimens. Small non-invasive and invasive carcinoma was found to be more common in fallopian tube than ovary. These findings are on the basis of complete sectioning of the ovaries and fallopian tubes in women who were at high risk of developing ovarian cancer, prophylactic salpingo-oophorectomy specimens. The study was conducted according to the SEE-FIM protocol. The non-invasive intraepithelial lesions are being labelled as serous tubal intraepithelial carcinoma (STIC) or high grade serous tubal intraepithelial neoplasia. They have cytological features almost identical to high-grade serous ovarian cancer and also show TP53 mutation, aberrant p53 protein expression, high proliferation indices and marked genomic instability ${ }^{5}$.

Epidemiologic studies of ovarian cancer depend on accurate tumours classification. Recent studies revealed that a huge percentage of mucinous carcinomas involving the ovaries were not primary, but metastatic lesions from elsewhere. The association between smoking and ovarian mucinous carcinoma is not clear because some smokingassociated mucinous carcinomas with their primaries outside ovary such as pancreatic cancer can behave in the same manner as primary ovarian mucinous tumours when they metastasize to the ovary creating diagnostic challenge. Rates of ovarian cancer increase proportionately with age. Differences in hereditary syndromes and differences in the pathogenesis of different types of ovarian carcinoma can be assessed with the help of varying age presentation among the sub groups. On average, younger women in their early reproductive age with invasive ovarian cancer will have a more favourable stage distribution and other prognostically favourable features. This helps in having a better prediction in outcome compared to others in elderly age group.

Ovarian carcinogenesis can broadly be classified into 2 entities: malignant transformation and peritoneal dissemination. It was presumed that benign, "borderline, " and malignant ovarian tumours contemplate successive steps in malignant transformation, irrespective of their type of differentiation like, serous, mucinous, endometrioid, or clear cell, by many authors till recently. According to many of the recent literatures the concept that ovarian carcinoma begins in the ovary and spreads sequentially from the ovary to the pelvis, abdomen, and then later to distant sites is being challenged. The old concept that ovarian cancer progress over time from well to poorly differentiated does not appear to be rational6.

About $2 / 3^{\text {rd }}$ of ovarian tumours occurs in reproductive age group, whereas incidence in children is less than $5 \%$. Natural history and response to treatment vary considerably from one group of tumours to others. As there are no screening tests for ovarian tumours and these tumours cannot be confidently distinguished from one another on the basis of their clinical, radiological or gross characteristics, it is important to determine the histological pattern of ovarian tumours to achieve optimum treatment response as prognosis depends on the degree of differentiation

From the latest researches, the various molecular alterations involved in serous carcinogenesis has been revealed. Now the pathologists have two different types of serous carcinoma. In the fourth edition of the WHO classification, low-grade serous carcinoma is the term used to label those serous neoplasms that are associated with serous borderline tumours/atypical proliferative tumours and include tumours which were previously categorized as grade 1 serous carcinoma. The term high-grade serous carcinoma is used to denote most of the malignant non-uterine serous carcinomas of the female genital tract previously classified as grade 2 or grade 3 serous cancers. This do not otherwise require additional grading. For several reasons, the consensus meeting held in Lyon before the publication of new classification system did not lead to settlement on a single, unifying concept for borderline tumours of the ovary4.

First, borderline serous tumours include a unique group of tumours that is biologically distinguishable from borderline tumours of other histological types. Borderline serous tumours can present at high stage, with or without nodal involvement. These are associated with a distinct, low, risk of transformation in time to low grade serous carcinoma. If they are adequately sectioned, in contrast, non-serous borderline ovarian tumours are restrained to the ovary and generally poses no risk for transformation.

Secondly, significant differences are existing among various gynaecological pathologists in association with borderline serous tumours in particular. Some pathologists have labelled the borderline serous tumours as benign and reported these lesions as atypical proliferative tumour, while others viewed that the borderline tumours terminology uniquely captures the true and the strange nature of these tumours. There are only very less literatures regarding malignant behaviour in well sampled tumours. This showed that the application of the terminology borderline tumours to the non-serous types is wrong. This has made many pathologists to favour the term atypical proliferative for these kinds of lesions. However, as the term border line has been used for long time, it has been preserved. Accordingly, both terminologies are accepted in the current (Fourth) edition of the WHO classification ${ }^{4}$.

\section{METHODS}

This is a retrospective study done in 3228 women who came to our hospital during the period January 2017- December 2018. All patients who had ovariotomy were included in the study irrespective of whether hysterectomy was done or not. All samples were collected from the operation theatre and preserved in buffered formalin. Representative sections were taken and stained with standard Haematoxylin and eosin stain. All the lesions were classified using WHO guidelines

\section{RESULTS}

A total of 3228 cases were studied among which 1536 (47.5\%) were normal. Among the remaining 1692 cases $73.8 \%$ cases were non neoplastic and $24.40 \%$ cases were benign. Malignant cases accounted for only $1.65 \%$ of cases. 


\begin{tabular}{|c|c|c|}
\hline Follicular cyst & 983 & $58.09 \%$ \\
\hline Lutein cyst & 157 & $9.27 \%$ \\
\hline Endometriotic cyst & 107 & $6.32 \%$ \\
\hline Hyperthecosis & 1 & $0.05 \%$ \\
\hline Ectopic gestation & 2 & $0.11 \%$ \\
\hline \multicolumn{2}{|c|}{ Table 1. Non-Neoplastic Cases } \\
\hline
\end{tabular}

Among the non-neoplastic cases, most common was follicular cyst accounting for $58.09 \%$ of the total cases followed by lutein cyst.

\begin{tabular}{|c|c|c|}
\hline Serous cystadenoma & 284 & $16.78 \%$ \\
\hline Serous cyst adenofibroma & 6 & $0.35 \%$ \\
\hline Mucinous cystadenoma & 53 & $3.13 \%$ \\
\hline Teratoma & 70 & $4.13 \%$ \\
\hline \multicolumn{2}{|c|}{ Table 2. Benign } \\
\hline
\end{tabular}

Serous cystadenoma accounted for almost $16 \%$ of the total cases.

\begin{tabular}{|c|c|c|}
\hline Papillary serous cystadenocarcinoma & 6 & $0.35 \%$ \\
\hline Granulosa cell tumour & 7 & $0.41 \%$ \\
\hline Yolk sac tumour & 1 & $0.05 \%$ \\
\hline Sclerosing stromal tumour & 1 & $0.05 \%$ \\
\hline Sex cord stromal tumour & 10 & $0.59 \%$ \\
\hline Brenner tumour & 3 & $0.17 \%$ \\
\hline Table 3. Malignant \\
\hline
\end{tabular}

Sex cord stromal tumours accounting for $0.59 \%$ cases was the commonest in malignant cases.
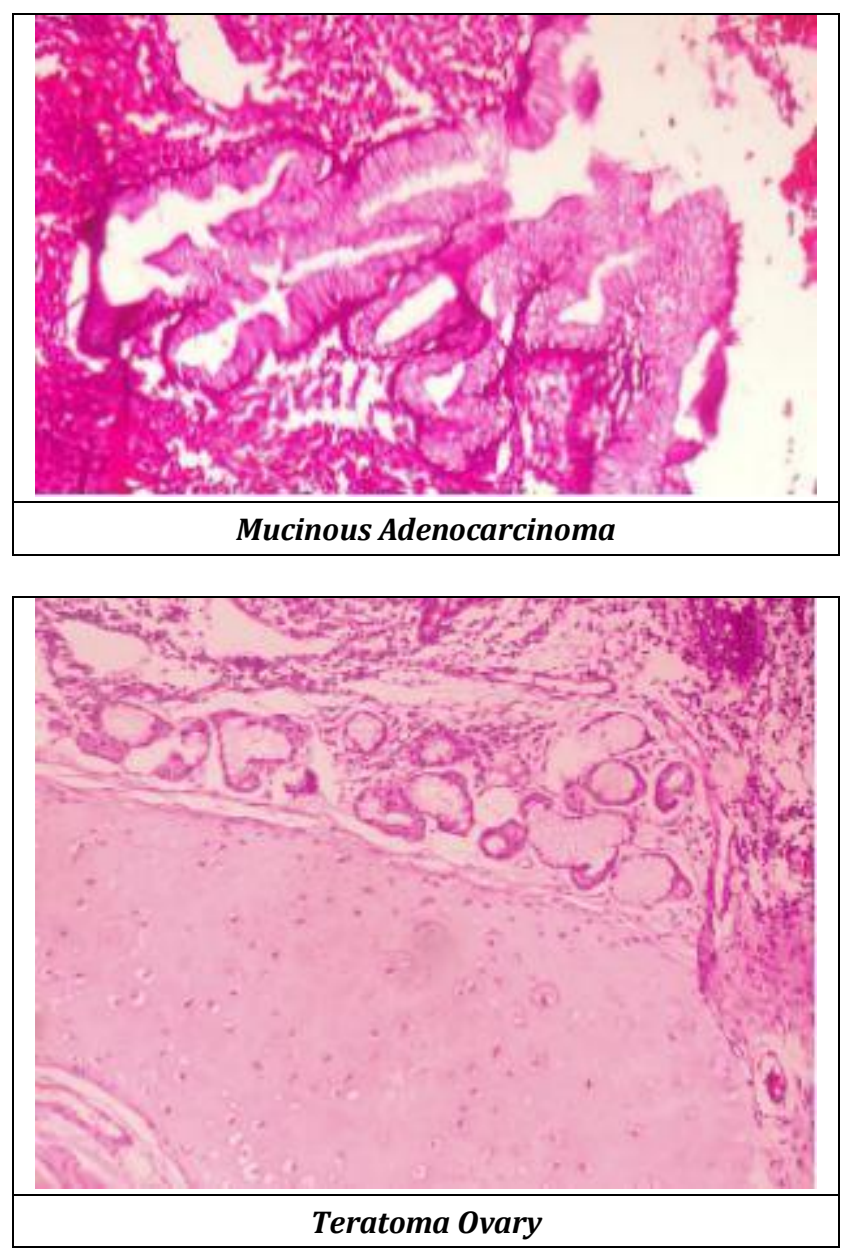

\section{DISCUSSION}

Although ovarian tumours are one the commonest entities their diagnosis often possess a challenge due to variety of pathologic conditions affecting the ovary. Thus, a detailed evaluation and expertise in morphology can help in improving the diagnosis. In our study, 1250 cases were non neoplastic lesion which accounted for $38.7 \%$ which was compatible with study done by Prakash et $\mathrm{al}^{2}$ which was $44 \%$ and Zaman et $\mathrm{al}^{7}$ which was $68.87 \%$. Follicular cyst was the most common non neoplastic lesion in our study which was $30.4 \%$ followed by lutein cyst and endometriotic cyst, $2.8 \%$ and $3.34 \%$ respectively which was compatible with studies done by Prakash et al and Maliheh et $\mathrm{al}^{8}$. Benign neoplastic lesion constitutes around 24.47 which was less than our comparative studies as we have included hysterectomy cases with attached ovary, where ovary didn't have any significant neoplasms. The percentage was shown much higher in studies done by Pachori et $\mathrm{al}^{9}$ which was $72.3 \%$ and Thakkar ${ }^{10}$ which was $84.5 \%$. Most of the tumours belonged to epithelial tumours category in our study which was $79.13 \%$ which was comparable to results of Krishna $\mathrm{M}$ et al ${ }^{11}$ which was $77 \%$ and Badge $S$ et al ${ }^{12}$ which was $77 \%$. In our study, 28 malignant cases were there which was all primary accounting for $100 \%$, similar observations were made by Bhagyalekshmi et $\mathrm{al}^{13}$ which was $98.5 \%$ and Thejasvani et al ${ }^{14}$ which was $98.9 \%$. Mature cystic teratoma constitutes about $15.8 \%$ of all tumours. Gupta S C et al 15 and Couto F et al 16 showed an incidence of $18.46 \%$ and $23.1 \%$ respectively. Among the malignant tumours Granulosa cell tumours accounted for $25 \%$ of cases which was compatible with the study done by Neha G et al ${ }^{17}$ which was $18.8 \%$.

\section{CONCLUSIONS}

Ovarian tumours include a wide spectrum of lesions. Emergence of borderline tumours with prognostic difference from benign and malignant counterparts has added a new dimension to the research in field of ovarian tumours. Correlation of clinical and radiological findings has assisted the pathologist to arrive at a definite conclusion in almost all cases. Even then, we may require the help of modern techniques like IHC and hormonal studies. Understanding in detail about the different lesions in the ovary, their prognostic implications and differences in the treatment is also crucial in diagnosis. A proper, complete and definite diagnosis helps the clinician to initiate the best conservative treatment available.

\section{REFERENCES}

[1] Fatima R, Sandhya M, Sowmya TS. Study of histomorphological pattern of ovarian neoplastic and non-neoplastic lesions. International Journal of Research in Medical Sciences 2017;5(5):2095-8.

[2] Prakash A, Chinthakindi S, Duraiswami R, et al. Histopatholological study of ovarian lesions in a teritiary care centre in Hyderabad, India: a retrospective 5 year study. International Journal of Advances in Medicine 2017;4(3):745-9. 
[3] Hawaldar R, Sodani S, Patidar E. Histopathological spectrum of ovarian tumours - a two year retrospective study. Indian Journal of Pathology and Oncology 2017;4(3):450-3.

[4] Kurman RJ, Carcangiu ML, Herrington CS, et al. WHO Classification of tumours of female reproductive organs. Fourth Edition. 2014.

[5] Kurman RJ, Ellenson LH, Ronnett BM. Blaustein's Pathology of the female genital tract. $6^{\text {th }}$ edn. Springer 2011.

[6] Rosai J. Female reproductive system. In: Ackerman's Surgical Pathology. $8^{\text {th }}$ edn. New York: Mosby - Year book Inc., 1996: p. 1473-539.

[7] Zaman S, Majid S, Hussain M, et al. A retrospective study of ovarian tumours and tumour-like lesions. J Ayub Med Coll Abbottabad 2010;22(1)104-8.

[8] Maliheh A, Yasmin S. Surgical histopathology of benign ovarian cyst: a multicentric study. Iranian J Pathology 2010;5(3):132-6.

[9] Pachori G, Meena US, Sunaria RK, et al. Histopathological study of ovarian tumours in Ajmer region. Int J Med Sci Public Health 2016;5(7):1400-03.

[10] Thakkar NN, Shah SN. Histopathological study of ovarian lesions. Int J Sci \& Research (IJSR) 2015;4(10):1745-9.
[11] Krishna M, Maurya G. Pattern of ovarian tumours and their age distribution in Kangra valley, Himachal Pradesh. Journal of Evolution of Medical and Dental Sciences 2015;4(61)10602-08.

[12] Badge SA, Gosavi AV, Sulhyan KR. Histopathological study of ovarian tumours. Indian Medical Gazette 2013: p. 345-51.

[13] Bhagyalakshmi A, Sreelekha A, Sridevi S, et al. Prospective study of histopathological patterns of ovarian tumours in a tertiary care centre. Int J Res Med Sci 2014;2(2):448-56.

[14] Vaddatti T, Reddy ES, Premalatha P, et al. Study of morphological patterns of ovarian neoplasm. IOSR Journal of Dental and Medical Sciences 2013;10(6):116.

[15] Gupta SC, Singh PA, Mehrotra TN, et al. A clinicopathological study of ovarian tumours. Indian J Pathol Microbiol 1986;29(4):354-62.

[16] Couto F, Nadkarni NS, Rebello MJ. Ovarian tumours in goa - a clinicopathological study. Journal of Obstetrics and Gynaecology of India 1993;43(3):408-12.

[17] Neha G, Anand AS, Annigeri C. Study of histomorphological spectrum of ovarian tumours. International Journal of Medical and Health Research 2017;3(10):12-20. 